\title{
SOBRE LA RELACIÓN ENTRE LOS «USOS SOCIALES»Y LAS «CREENCIAS» EN LOS ÚLTIMOS AÑOS DE ORTEGA
}

\section{REGARDING THE RELATION BETWEEN «SOCIAL USES»AND «BELIEFS»IN ORTEGA'S FINAL YEARS}

\author{
Juan MAnuel Monfort PRAdes* \\ Universidad Cardenal Herrera CEU San Pablo de Castellón
}

\begin{abstract}
Resumen: No cabe duda de que tanto el concepto de "uso" como el de "creencia» ocupan lugares muy destacados en la filosofía de Ortega. En un principio cada uno responde a diferentes inquietudes del madrileńo, ya que si la «creencia» está vinculada principalmente a la percepción de la realidad, el de «uso» es la clave de toda la sociología de Ortega. Ello haría pensar que se tratan de conceptos relacionados con ámbitos diferentes entre los que no cabría establecer conexión ninguna. Ahora bien, existen autores que los identifican y autores que insisten en la confusión que ello supone. Este escrito intenta arrojar un poco de luz a esa problemática.
\end{abstract}

Palabras Clave: creencia, idea, usos sociales, cultura, vida.

AвSTRACT: There is no doubt about the crucial role of the concepts of «social use» and "belief» in Ortega's philosophy. Each one is an answer to different intellectual issues that Ortega outlines in his works. If, on one hand, «belief» is related to Ortega's epistemology, on the other hand, «social use» is bonded with his sociology. Even if both concepts are very different at first sight, they could be regarded as the same as some experts actually do. This text tries to shed some light on the issue of the equivalence of these important concepts.

KeYwords: belief, idea, social uses, culture, life.

* Profesor Asociado, idjmal@hotmail.com, C/Tirant Lo Blanc, 1, 3. p.9, 12100, Castellón. 
Cuando un lector se aproxima a la obra de Ortega, percibe con claridad que tanto el concepto de "uso" como el de "creencia» ocupan lugares muy destacados en su filosofía. En un principio, parece ser que cada uno responde a diferentes inquietudes del madrileño, ya que si la creencia aparece vinculada principalmente a la percepción de la realidad, el de uso es la clave de toda la sociología de Ortega.

Ello haría pensar que se trata de conceptos que hacen referencia a ámbitos diferentes, por lo que no cabría establecer entre ellos una gran conexión y, menos aún, una identificación. Ahora bien, existen intérpretes, como veremos en esta exposición, que los llegan a identificar, a la vez que otros insisten en el diferente significado que posee cada uno. En definitiva, ¿es lo mismo el «uso» y la "creencia» en Ortega? ¿Todos los «usos» son «creencias»? ¿Son todas las «creencias» también «usos»? Este escrito intenta arrojar un poco de luz a esa problemática, iluminando así la relación entre ambos conceptos. Para ello concentraremos nuestra atención, en primer lugar, en los textos de Ortega más relevantes referidos a estos temas, posteriormente, presentaremos los trabajos más significativos escritos por intérpretes que se han acercado a este asunto. Finalmente, presentaremos una serie de conclusiones.

El concepto de «uso» aparece en numerosas obras de Ortega, no sólo en su madurez, sino también en los inicios. Cierto es que el desarrollo del mismo no llega hasta El hombre y la gente y que antes de ese trabajo el «uso» no es nada más que la costumbre, el tópico, algo contra lo que Ortega ha luchado desde su juventud por su irracionalidad y falta de vitalidad. «No los abusos son lo peor de España, sino los usos» (O.C. I, p. 837), afirma en «La nación frente al Estado» de 1915.

Cuando se procede a la lectura de La rebelión de las masas, los «usos» aparecen en diferentes lugares y se aprecia una profundización del pensamiento de Ortega en cuanto a dicho concepto. Debemos tener en cuenta para ello los momentos en los que se van componiendo las diferentes partes de la obra. En la lección cuarta de 1929 se lee:

De ordinario rehuimos palpar esa pulsación pavorosa que hace de cada instante sincero un menudo corazón transeúnte; nos esforzamos por cobrar seguridad e insensibilizarnos para el dramatismo radical de nuestro destino, vertiendo sobre él la costumbre, el uso, el tópico -todos los cloroformos (O.C. IV, pp. 398-399). 
Se puede decir que Ortega mantiene en este párrafo el concepto de «uso» que maneja desde su juventud, es decir, el «uso» no es más que la costumbre, y ésta tiene para Ortega, en este contexto, un marcado sentido negativo. En cambio, en el anexo titulado "En cuanto al pacifismo» de 1937 se aprecia ya una idea más elaborada que dista bastante de la anterior:

La convivencia, sin más, no significa sociedad, vivir en sociedad o formar parte de una sociedad. Convivencia implica sólo relaciones entre individuos. Pero no puede haber convivencia duradera y estable sin que se produzca automáticamente el fenómeno social por excelencia, que son los usos —usos intelectuales u «opinión pública», usos de técnica vital o "costumbres», usos que dirigen la conducta o «moral», usos que la imperan o «derecho»-. El carácter general del uso consiste en ser una norma del comportamiento -intelectual, sentimental o físico- que se impone a los individuos, quieran éstos o no (O.C. IV, pp. 515-516).

Como puede apreciarse, se perciben ahora algunos elementos nuevos referidos al concepto comentado, como puede ser su carácter impositivo. No cabe duda de que entre ambos textos de La Rebelión de las masas Ortega se ha decidido a desarrollar el concepto de «uso». ¿Qué ha sucedido en esos años? Sencillamente que Ortega ha puesto en marcha un gran proyecto llamado El hombre y la gente, título que refiere a un conjunto de escritos de diferentes años y de diferentes contenidos, entre los conceptos más destacados de dicha obra se encuentra, sin duda, el de "uso». Tras la publicación de las nuevas Obras completas de Ortega, cuando se habla del trabajo El hombre y la gente, hay que matizar a cuál de de las obras se refiere el que lo cita. Hasta ahora la referencia publicada era el trabajo que preparó Paulino Garagorri, pero en estos tiempos se ha desvelado, gracias al equipo de investigación de las obras, la complejidad y génesis de ese trabajo. De 1934 se conserva la conferencia en Valladolid que lleva por título «El hombre y la gente», en 1936 otra conferencia en Rotterdam con el mismo nombre, 1939-1940 es el año en que Ortega da un primer curso de varias lecciones con ese mismo título y, finalmente, entre 1949 y 1950 procede a impartir otro conjunto de lecciones. La novedad no es tanto el conocimiento de los textos, como la publicación encuadrada en el resto de la obra de Ortega. Las «notas a la edición» de los volúmenes IX y X de las Obras completas de Ortega se han convertido en referencias imprescindibles para comprender este texto.

Por su parte, el de creencia, también es un concepto que admite una evolución, aunque cabe ańadir que su problematicidad y complejidad dentro de 
la obra de Ortega es mayor que la del «uso». En 1915-1916 en Sistema de la psicología y Los problemas actuales de la filosofía Ortega desarrolló un concepto de creencia vinculado con la fenomenología de Husserl que Lorenzo Alquezar estudio con detenimiento (Lorenzo Alquezar, R., 1999, pp. 136 y 137). Se propone Ortega en estos trabajos, nada más y nada menos, que hacer una filosofía de la ciencia con un estilo propio, superando a los clásicos y con un objetivo claro: perseguir los fundamentos de la psicología en cuanto ciencia. Precisamente son los fundamentos, más que la psicología en sí misma de lo que va a hablar Ortega, y para aclararlos no tiene otra que profundizar en el papel de la ciencia, en sus objetivos y en sus supuestos. En este contexto propone Ortega que la «creencia» es la declaración de que lo creído vive por sí mismo, independientemente de mi acto de creer, ni nace ni muere con éste. «La creencia, en suma, es la conciencia de que algo es -es independiente de esta mi conciencia» (O.C. VII, p. 514), por lo que podría afirmarse que el ser no es sino el correlato del creer. Se puede decir que la creencia en 1915 tiene, según los textos de Ortega, un carácter noético fundamental: es posición o tesis de realidad. Más tarde, lejos ya del contexto fenomenológico que envuelve a Ortega en los años del Sistema de la psicología, aparece de nuevo el concepto en Ideas y creencias llegando a su planteamiento definitivo.

Sin dejar de prestar atención a lo expuesto hasta ahora, conviene adentrarse en el análisis de los conceptos de "uso" y «creencia» en los trabajos orteguianos que se han destacado como fuentes principales, donde Ortega lleva a cabo sus reflexiones más profundas sobre ellos: El hombre y la gente e Ideas y creencias.

\section{EI «uso social» en El hombre y la gente}

¿Cómo puede resumirse el contenido que El hombre y la gente ofrece como propio de la idea de «uso social»? Se intentará destacar a continuación la serie de elementos que caracterizan este concepto.

En primer lugar, el «uso social» es un acto ejecutado por un ser humano, pero los seres humanos al nacer ya se encuentran con un conjunto de usos que le acogen. Dice Ortega:

Los usos están siempre ahí, quiero decir que el hombre al nacer en un lugar de la tierra y hallarse, por tanto, en el mundo, encuentra que en ese lugar de la tierra están, junto a minerales, vegetales, animales y los otros 
hombres, usos con los cuales tiene que contar, quiera o no; que, por lo tanto, son una realidad exterior a él, que influyen en él (O.C. IX, p. 350).

Los usos son realidades exteriores al ser humano y entre ellos se da una influencia mutua: los usos ejercitan una presión sobre el hombre, pero éste también la lleva a cabo sobre los usos, llegando a producir cambios o eliminaciones de usos con el paso del tiempo. Aunque el uso es ejecutado por un ser humano, cabe destacar que tal uso no ha sido inventado por su ejecutor, ni siquiera lo entiende. Más bien repite lo que otros hacen y, por ello, puede decirse que el uso tiene un origen extraindividual, el autor no es nadie determinado. A la vez, el yo no ejecuta el acto por espontánea voluntad, sino que es en muchas ocasiones impuesto. En definitiva, es un acto al que le faltan dos caracteres clave de toda acción humana: originarse intelectualmente en el sujeto que lo hace y engendrarse en su voluntad. Por ello más que un actuar humano es un actuar mecánico.

En segundo lugar cabe destacar que el uso aparece como una amenaza, pues negar el saludo a alguien, como recuerda Ortega en su famoso ejemplo, puede conllevar una coacción contra mí (en otras épocas implicaba ser retado a un duelo). No podemos pues dejar de hacerlo, de lo contrario puede decirse de esa persona que es un maleducado, en el sentido de que no respeta las normas básicas de convivencia que la gente, con el tiempo, ha impuesto y en su violación se siente ofendida la sociedad en pleno.

La tercera característica del uso que será destacada es haber perdido el sentido que un día tuvo, ya que con el tiempo se convierten en acciones desalmadas, vacías de la fuerza vital que les llevó a la existencia. Recordando las palabras de Ortega, de la misma forma que el saludo en cuanto tal ha perdido su sentido a lo largo del tiempo, también al uso

le es constitutivo haber perdido su sentido, por tanto, haber sido un tiempo acciones humanas interindividuales e inteligibles, acciones con alma y haberse luego vaciado de sentido, haberse mecanizado, automatizado, como mineralizado, en suma, desalmado. Fueron auténticas vivencias humanas que luego, por lo visto, pasaron a ser supervivencias, a ser humanos petrefactos. Por eso hablo de mineralización (O.C. X, p. 273).

Una cuarta característica de los usos es que estos no son una cuestión de acuerdos sociales, la supresión o creación excede a las posibilidades de una persona y es un proceso lento. Afirma Ortega: 
Desde el instante en que un individuo tuvo la idea creadora -sólo los individuos crean-, la idea creadora del nuevo uso, hasta que éste llega a ser, en efecto, uso vigente, institución -todo uso es institución-, tiene por fuerza que pasar mucho tiempo. Y en el lapso de este largo tiempo que tarda en formarse un uso, la idea creadora que en su hora inicial tuvo pleno sentido, cuando se hace usual, cuando se hace modo social, en suma, uso, ha empezado ya a ser anticuada, a perder el sentido que tuvo, a ser ininteligible. Lo cual -conste, nótenlo- no daña, por lo pronto, al uso; porque lo que hacemos porque se usa no lo hacemos porque nos parezca bien, porque lo juzguemos razonable, sino mecánicamente lo hacemos porque se hace y, más o menos porque no hay otro remedio (O.C. X, p. 281).

Otra característica destacable es que son clasificables. Ortega diferencia dos grandes tipos de usos: débiles y fuertes. Los primeros son difusos y débiles, son las costumbres del comer, el vestir, las órdenes del trato social corriente, el hablar de la gente, la lengua en sí misma y la opinión pública. En cambio existen otros que Ortega llama usos fuertes, rígidos o prescripciones, entre ellos se encuentran los económicos, el Estado o política y el Derecho, este último es reconocido por Ferrerio Lavedán como el uso fuerte por excelencia (ver Ferreriro Lavedán, 2005, pp. 219-256). En los primeros se ofrece cierto margen de maniobra a los hombres, los segundos tienen un carácter fuertemente coactivo y violento.

En sexto lugar, los usos son de gran utilidad para el ser humano, al menos en tres sentidos, según el curso de 1939-40: amortiguan las relaciones sociales, conforman un plan de vida actual para aquellos incapaces de imaginarlo y, por último, ahorran tiempo y energías.

En séptimo y último lugar, los usos sociales, por la presión que ejercen sobre las personas, son llamados por Ortega vigencias sociales. Lo que actúa de ellos no es más que su presión o coacción sobre los hombres, no necesitan que nadie se preocupe en sostenerlos. "Cuando algo es uso no depende de la adhesión de los individuos sino que precisamente es uso porque se impone a ellos. Merced a esto, todo lo social es realidad diferente de lo individual» (O.C. X, p. 320).

\section{La «creencia» en Ideas y creencias}

Por otra parte, ¿cómo plantea Ortega el concepto de «creencia» en Ideas y creencias? Veamos por separado cómo procede en los dos capítulos de la obra. 
En cuanto al capítulo primero podemos destacar las siguientes características de las «creencias»:

En primer lugar, el punto de partida de la reflexión sobre las "creencias» es la vida de cada uno, vivir es tener que habérselas con el mundo y consigo mismo, pero éstos aparecen interpretados, no en su realidad desnuda. Las creencias constituyen el continente de nuestra vida y no tienen el carácter de contenido particular dentro de ésta. Son ideas que somos, no ideas que tenemos, como dice Ortega: «con nuestras creencias estamos inseparablemente unidos. Por eso cabe decir que las somos» (O.C. V, p.667). Al contrario que las creencias, las ideas son producidas y sostenidas por cada uno. Con las creencias no hacemos nada, sólo estamos en ellas, vivimos de ellas. Si la idea se tiene, en la creencia se está, nos tiene y nos sostiene. Hay ideas con que nos encontramos y creencias en que nos encontramos que están ahí antes de nuestro ejercicio intelectual.

En segundo lugar, la función de la creencia es sostenernos y ser el continente de nuestra vida, operan de forma latente en nuestro fondo cuando decidimos pensar en algo. Son la tierra firme sobre la que nos afanamos, pero actúan sin ser nosotros conscientes, poniendo delante lo que para nosotros es la realidad misma. Realidad es precisamente aquello con que contamos, queramos o no, lo que nosotros no ponemos sino que encontramos, de la misma manera que el urdimbre sostiene la trama.

La tercera característica destacable del primer capítulo de Ideas y Creencias es la posibilidad de que en las creencias también se abran agujeros de duda. Cuando esto sucede, se está como cayendo en un abismo, como la muerte en vida, es vivir en el instante de un terremoto, en lo inestable como tal. Esos agujeros de duda son el lugar donde anidan las ideas. Con ellas se trata de paliar la duda, creando mundos interiores de ideas fruto de la fantasía que procuran recuperar la seguridad perdida tras el ocaso de una creencia.

El segundo capítulo de Ideas y creencias nos ofrece otros aspectos de la creencia que conviene recapitular para obtener una visión conjunta y más completa de este concepto:

Primero, las creencias son todas aquellas cosas con que absolutamente contamos, aunque no pensemos en ellas. No las cuestionamos porque las damos por sentadas y nuestra vida en todo momento está montada sobre un repertorio enorme de creencias parejas. 
Segundo, hay situaciones y cosas ante las que nos encontramos sin creencia firme, nos encontramos en la duda y no tenemos más remedio que hacernos una idea, una opinión sobre ellas. Realidad plena no es sino aquello en que creemos por lo que las ideas no son la realidad auténtica. Las ideas tienen una misión ortopédica, actúan donde una creencia se ha roto.

Tercero, las creencias no han sido las mismas a lo largo de la historia. La realidad de la Tierra ha variado considerablemente para los hombres: si hoy es un astro que sostiene, antes era la diosa Deméter. Ello supone reconocer que la realidad de la Tierra es un enigma y que al quitar las capas de ideas que se han sedimentado sobre ella no quedan más que facilidades o dificultades para nuestra vida.

En cuarto y último lugar, según lo dicho, vivir es encontrarse viviendo en lo enigmático, a esa situación reacciona el hombre con su aparato intelectual, con la imaginación crea los mundos interiores y ofrece una cierta claridad y seguridad a su vivir. Algunas de esas ideas, con el tiempo se consolidan en creencias. La creencia es certidumbre en que nos encontramos sin saber cómo ni por dónde hemos entrado en ella, de ahí que pueda decirse que toda creencia es recibida.

\section{Una reflexión sobre el concepto de «creencia»}

El catedrático de la UNED Javier San Martín hace una profunda reflexión sobre estos dos capítulos que conforman la obra de Ortega Ideas y creencias. Insiste en que cada capítulo promociona un concepto diferente de creencia según se lee en su trabajo "IIdeas y creencias". Comentario a un texto de Ortega» (San Martín, 1998, pp. 213-245). Veamos qué razones le permiten realizar tal afirmación.

En primer lugar es clave la procedencia de los diferentes capítulos. Pese a que el texto se publica en 1937, se escribió en 1934 y el contexto es clave. Ortega había impartido varios cursos muy importantes en los ańos anteriores: En torno a Galileo en 1933 y Unas lecciones de metafísica en 1932. Ambos cursos son imprescindibles para comprender Ideas y creencias.

El capítulo primero es un ensayo de descripción de la estructura de la vida humana a partir de tres situaciones: creencia, idea y duda. Expone Ortega un concepto de creencia a partir del esquema creencia-duda-idea, aparece así una 
idea de creencia más epistemológica o metafísica: creencia es fundamentalmente la pura realidad, nos pone delante de la realidad misma frente a la realidad virtual que representan las ideas. Esta vertiente del concepto de creencia es la que Ortega desarrolló en Sistema de la psicología y ahora presenta de nuevo bajo el influjo de Unas lecciones de metafisica impartidas previamente a la redacción de Ideas y creencias.

El capítulo segundo aparece como un diagnóstico de la época contemporánea al tratar sobre las ideas y su valoración como producto intelectual. Comenta Ortega la crisis del siglo XX en torno a la razón y la rebarbarización del hombre como resultado de la misma. Expone Ortega un concepto de creencia a partir del esquema duda-idea-creencia. Así aparece un concepto de creencia en un sentido más histórico y de crítica cultural, en un sentido más sociológico o político. Si el primer concepto se caracteriza por la confianza en la realidad, el segundo se caracteriza por entender la creencia como un modo de vivir a crédito, no en la realidad misma. La realidad en la que se vive se debe a otros hombres, la realidad en la que se cree no es la auténtica, hay que vivir del crédito de quienes antes interpretaron la realidad. Ésta no es más que un enigma. De alguna manera el concepto de creencia se separa así de la gran carga epistemológica que Ortega le daba en el contexto anterior. Ortega parte de la duda que sugiere una realidad enigmática, a la cual se responde con las ideas y estas al solidificarse se convierten en creencias.

Con todo esto, el concepto de creencia adquiere tintes diferentes a los anteriores. Lo importante no es que nos pone ante la realidad, la cual se convierte en un enigma, sino que las creencias son ideas petrificadas que, una vez perdido el sentido que les llevó a ver la luz, quedan en la sociedad como un substrato desde el cual se vive. Es una realidad desde la que se vive, pero ese aspecto ya no es el principal, la clave no es la creencia como un concepto original, sino que las creencias son las ideas que con el tiempo se consolidan. Cristaliza un concepto que Ortega va a poder aprovechar para estudiar el desarrollo de la cultura y de la sociedad.

Es posible que estas dos formas de explicar el concepto de creencia tengan más repercusiones de las esperadas. La cuestión que se plantea es la siguiente: ¿es posible que estas dos vertientes del concepto tengan diferentes derivaciones? A mi modo de ver, creo que la segunda vertiente del concepto de creencia, más vinculada a la sociología y a la crítica cultural, la va a conectar Ortega con el concepto de "uso social», de forma que ambos conceptos pueden considerarse 
sinónimos si por «creencia» entendemos tan sólo el segundo significado planteado. Si en un primer momento "uso» no significaba mucho más que costumbre, a partir de ahora, adquiere el carácter de creencia en el segundo sentido expuesto y de ahí su enriquecimiento y su profundidad en las exposiciones de El hombre y la gente. Ahora bien, no podemos olvidar que, en realidad, creencia tiene un sentido más amplio cuando se cuenta con esa vertiente epistemológico-metafísica que sigue la estela del Sistema de la psicología y que es, al fin y al cabo, el primer sentido que Ortega le dio.

¿Qué razones existen para llegar a esta conclusión? Comparemos brevemente los conceptos de uso y de creencia. Partiremos de la síntesis que José Lasaga (Lasaga Medina, 2003, p. 148) hace de la creencia frente a la idea en su trabajo José Ortega y Gasset (1883-1955). Vida y filosofía, destacando los siguientes aspectos de la creencia: con las creencias se cuenta, tienen carácter de realidad, son el continente de la vida humana, son latentes o inconscientes, tienen vigencia social y, por último, son colectivas y heredadas. Esta serie de características de las creencias nos va a permitir hacer una comparación con el «uso» a partir de unos textos de Ortega. Veamos una a una las características que propone Lasaga y contrastémoslas con textos orteguianos.

Dice Ortega en El hombre y la gente que el uso o la vigencia social «no se nos presenta como algo que depende de nuestra individual adhesión, sino que, por el contrario, indiferente a nuestra adhesión, está ahi, tenemos que contar con ella y ejerce, por tanto, sobre nosotros su coacción» (O.C. X, p. 320). Parece pues que con los usos contamos de la misma manera que contamos con las creencias.

En segundo lugar, dice Ortega a los argentinos: «los usos que como una atmósfera envuelven a ustedes y de que están ustedes transidos hasta la médula de su hombría, traen para ustedes la clara contraseña de ser los usos de la Argentina» (O.C. IX, p. 426). Marcan el carácter personal y son la base de humanidad a partir de la cual se construye la vida de cada uno, por ello puede decir Ortega que permiten "vivir desde un cierto nivel de humanidad ya progresada» (O.C. IX, p. 355). Los usos nos envuelven y desde ellos se vive, es decir son, como las creencias, el continente de la vida humana.

El filósofo madrileño, al hablar de las opiniones como usos establecidos, dice: «y establecidos quiere decir que no necesitan del apoyo y sostén por parte de individuos o grupos determinados sino que, al revés, se imponen a todos, ejercen sobre todos su presión. Esto es lo que me lleva a denominarlas «vigencias» 
(O.C. X, p. 319). Es decir, considera que, tanto los usos como las creencias, poseen el carácter de vigencia social.

En cuarto lugar, veamos estas palabras de Ortega sobre los usos: «ciertamente, no hay colectividad, no hay sociedad sin usos. Pero los usos fueron primero invención de un individuo; luego, cultivo, desarrollo de esa invención por un grupo particular de individuos a quienes esa idea conquistó. Sólo después se convierten en vigencia social, en uso» (O.C. IX, p. 358). De la misma manera que las creencias, los usos no son fruto solamente de un individuo, sino que son fruto de un trabajo colectivo a través del cual adquieren solidez.

Las creencias también son heredadas, un aspecto que precisamente Ortega declara que el uso posee sin lugar a dudas: «El hombre — como dice Goethe- no es sólo descendiente sino heredero, tiene siempre -más o menos, alguna herencia, es hijo de algo. El hombre es, de nacimiento, hidalgo. Todo un pasado humano está ahí, preparado por la sociedad, preparado en forma de usos, que disecan y condensan todo ese enorme trabajo, esa larga continuidad de esfuerzos humanos» (O.C. IX, p. 355).

Acabamos de hacer referencia a cinco de los siete caracteres que Lasaga presenta de la creencia, y no parece que haya discusión sobre su paralelismo con los caracteres que se aprecian en los usos. ¿Qué sucede con los dos que faltan? Los dos caracteres reservados imprimen en el concepto de creencia su carácter más epistemológico: el ser "carácter de realidad» y el ser «latentes», por ello no se reflejan con facilidad en el uso social. Ahora bien, «uso es aquel hacer nuestro que hacemos no por gusto ni por cuenta propia — sino que lo hacemos simplemente porque se hace» (O.C. IX, p. 393). Ello denota una cierta anonimidad del uso que, junto a su efecto coactivo, le permiten tener un cierto carácter de «latencia» aunque no coincida ésta con el carácter de inconsciencia con que se presenta la creencia. Sin embargo, Ortega utiliza con el uso el mismo ejemplo que en Meditaciones del Quijote le sirvió para distinguir lo latente y lo patente, el ejemplo del bosque: «La coacción, como todo lo social, parece primero no consistir sino en lo que hacen con nosotros los demás individuos -porque lo social se oculta espontáneamente tras de los individuos como el bosque tras de los árboles y como al labriego de Poitiers la altura de las casas no le dejaba ver la ciudad» (O.C. IX, pp. 363-364). Por lo que se refiere al carácter de realidad, parece que en ningún momento Ortega otorga a los usos el carácter de realidad primordial que caracteriza a la creencia desde Sistema de la psicología, ya que, en 
el contexto de los usos, la realidad primordial se vuelve un enigma, como bien se puede comprobar en el segundo capítulo de Ideas y creencias.

Ante estos datos sólo caben dos posibilidades: o la "creencia» y el «uso» pueden identificarse en algún sentido, o "creencia» $\mathrm{y}$ «uso», pese a sus parecidos, son conceptos diferentes y su identificación sólo conlleva confusiones. Ambas posturas pueden reconocerse en dos autores que pueden considerarse autoridades en esta materia: Ciriaco Morón Arroyo y Jorge García-Gómez. El primero rechaza la posibilidad de identificarlos, mientras que el segundo entiende que coinciden. Veamos ambas posturas por separado e intentemos llegar a unas conclusiones.

\section{4. ¿Es el concepto de «creencia» sinónimo del «uso social»?}

Ciriaco Morón Arroyo en El sistema de Ortega y Gasset desarrolla esta cuestión a partir de las reflexiones de Julián Marías en torno a las «vigencias sociales» o «usos sociales» (ver Marías, 1969, pp. 230-310):

$\mathrm{Al}$ analizar el concepto de vigencia dado por Marías, hemos descubierto como fórmula definitoria el contar con; lo mismo encontramos en la definición orteguiana de creencia; la fórmula tiene distinto sentido aplicado a una o a otra: en las creencias, el contar con es siempre de signo positivo, es siempre estar identificado con ellas. Los contenidos de la creencia pueden ser socialmente vigentes o no; si el contenido de mi creencia se opone a lo socialmente vigente, tengo que contar con ello, pero en el plano de las ideas tengo que ocuparme de ello, porque me es problemático.

De esta comparación se deduce que mientras lo vigente es algo que habría que definir en términos puramente funcionales, estrictamente sociológicos, el tema de la creencia tiene implicaciones psicológicas y epistemológicas que le hacen un problema distinto. La subrepticia equiparación o correlación de creencias y vigencias, no conduce más que a confusiones (Morón Arroyo, 1968, p. 279).

Pese a los parecidos que pueden apreciarse entre los conceptos, la opinión de Morón Arroyo es la de reservar cada concepto para un ámbito de estudio diferente. La distancia entre ambos está marcada por las implicaciones psicológicas y epistemológicas de la creencia y ello obliga a distinguirlos si no se quiere crear graves confusiones en el estudio de la obra de Ortega. 
Jorge García-Gómez en «Las creencias y los usos» (García Gómez, 2009, pp. 143-158) parece tomar una postura distinta. El profesor emérito de la Long Island University presenta las creencias en sentido pragmático, pero ¿qué significa esto? Incidir principalmente en lo que la creencia tiene de servicio para la acción humana, pues las creencias

sirven para determinar la acción, en el sentido de posibilitarla, ofuscarla u obstaculizarla. Conforme a esto, las transformaciones históricas decisivas serían los correlatos de los cambios en las creencias más primitivas. Pongámoslo aún de otra manera: las creencias constituirían así la fuente suprema de autoridad práctica por la cual confirmar, a nivel inmediato, la validez o la ilegitimidad de nuestras empresas o iniciativas (García Gómez, 2009, p. 144).

Tras esta presentación de la idea de creencia, más adelante vuelve a insistir en este carácter clave de la misma:

Las creencias ni se adoptan ni surgen libremente, pues dotan de última dirección a la vida al encontrarnos sua sponte en ellas y serlas, llevándolas a la espalda y mirando hacia delante, por así decirlo. Es por ello que afectan todas las esferas de la actividad vital, ya sea teórica o práctica, y por consiguiente «determinan» la posibilidad y la producción de las ideas. Sería entonces más exacto decir que las ideas son como "modificaciones» de las creencias, y que únicamente constituyen éstas la «sustancia» de la vida como tal (García Gómez, 2009, p. 147).

El concepto que García-Gómez propone de creencia en este trabajo es principalmente práctico, por ello en el segundo apartado del ensayo puede relacionarlo con el de uso social que también es un concepto eminentemente práctico. Afirma que las creencias son un tipo de uso, los usos de índole intelectual que son considerados los principales por el autor:

Las creencias se distinguen de los demás usos por ser de índole «intelectual» y constituir así un saber que -como ya se ha insistido repetidamentenos pone sin mediación alguna en la realidad, lo que nos permite estar o, mejor aún, hacer nuestra morada o vivienda en la circunstancia en cuanto mundo. Como todo uso, una creencia es una acción que tiene efecto en mí, pero de modo tal que éste no se origina ni en mi voluntad ni en mi 
concepción ni en ninguna dimensión de mi espontaneidad (García Gómez, 2009, p. 151).

El aspecto de las creencias-usos que se destaca es el de ser residuos históricos o sedimentaciones de antiguas acciones humanas: «Las creencias, en tanto y en cuanto son usos, operan en nosotros como residuos históricos o sedimentaciones resultantes de antiguas acciones humanas en el sentido riguroso del término, o sea, en el de actos que se originan en alguien por razones propias» (García Gómez, 2009, p. 152).

En definitiva y volviendo a insistir en un sentido pragmático de la creencia como base de la identificación con los usos afirma:

Las creencias son el fundamento último de nuestra conducta social, o sea, de lo que hacemos, sentimos y pensamos en cuanto miembros de una sociedad, pero no como agentes responsable de nuestra vida, o sea, como aquellos que se proponen establecer relaciones propiamente interpersonales (García Gómez, 2009, p. 153).

Queda patente en los textos citados que para el profesor emérito de la Universidad de Long Island, el concepto de uso y el de creencia pueden identificarse si la creencia se toma desde el punto de vista pragmático. ¿Cómo conciliar esta postura con la de Morón Arroyo? ¿Sucede acaso que uno de ellos ha caído en un malentendido?

Parece claro que los usos, en su sentido más común según Ortega, son creencias, pero ¿toda creencia es uso social? Todo indica que la respuesta es negativa, ya que la creencia, si bien envuelve al concepto de uso, también lo excede.

\section{Conclusiones}

Pienso que existe una solución en la que los dos intérpretes de Ortega pueden tener razón, lo que nos permitirá recoger todo lo expuesto y plantear una serie de conclusiones finales.

El trabajo de Javier San Martín y su distinción de dos conceptos de creencia en Ideas y creencias aparece como un descubrimiento de gran importancia para comprender la relación entre «creencias» y «usos sociales». Plantea la existencia 
de dos conceptos de creencia: uno epistemológico-metafísico y uno de carácter histórico-pragmático.

Estos dos conceptos de creencia siguen caminos diferentes en los años posteriores. Mientras que Ortega sigue utilizando el concepto de creencia en los dos sentidos citados, el concepto de uso social da un giro de gran calado en su filosofía. El «uso» deja de ser una mera tradición y se enriquece con ese segundo concepto de creencia más histórico-pragmático, el cual permite a Ortega desarrollar su sociología y su crítica de la cultura. Esto lleva a una posible identificación de los conceptos de uso y creencia, aunque no cabe olvidar que la creencia tiene un carácter epistemológico-metafísico que el de uso no posee. ¿Por qué se puede realizar esta identificación? Dos razones: la primera es que coinciden en sus características definitorias principales, y la segunda es que las razones para no llevarla a cabo tienen que ver con el carácter epistemológico de la creencia, el cual no sería un problema si se tiene en cuenta la distinción de Javier San Martín, ya que quedaría para el concepto de creencia en un sentido más amplio.

En consecuencia ¿pueden identificarse «usos» y «creencias»? Si y no. Si se hace debe insistirse que es la vertiente pragmática o histórica del concepto de creencia la que se relaciona con el uso. De lo contrario podrían crearse confusiones. Si se opta por no llevar a cabo tal identificación, no es porque sean conceptos completamente diferentes, sino porque conviene destacar que la creencia tiene una vertiente epistemológica desde 1915, y es posible que ésta pudiese quedar relegada al olvido, ya que el concepto de uso no está planteado en esta clave.

Teniendo en cuenta estas reflexiones, tanto Morón Arroyo como GarcíaGómez tendrían razón. El primero de ellos, porque asume la creencia principalmente en sentido epistemológico y no puede conjugarlo con el de uso. El segundo, porque entiende la creencia en su vertiente pragmática y, en ese sentido, son identificables.

¿Qué parece ser lo más adecuado: identificar o distinguir? Todo indica que lo menos confuso es evitar la utilización de "creencia» de forma sinónima a la de «uso» ya que no son completamente equivalentes. Ahora bien, debe quedar claro que en el fondo los «usos» no son más que "creencias», o como Ortega dice en Historia como sistema, "creencias colectivas»:

Por lo demás, la instauración de un nuevo uso — de una nueva «opinión pública» o "creencia colectiva», de una nueva moral, de una nueva forma 
de gobierno-, la determinación de lo que la sociedad en cada momento va a ser, depende de lo que ha sido, lo mismo que la vida personal (O.C. X, p. 69).

Pese a lo expuesto en esta breve reflexión, cabe notar que los conceptos de uso y de creencia poseen una complejidad mayúscula en la filosofía de Ortega. En sus desarrollos las concepciones fluctúan y no resulta fácil delimitar sus significados, de ahí que la cuestión de identificar o no a ambos sea un asunto a debatir que puede sugerir diferentes posturas de opinión.

\section{Bibliografía}

Ferreiro Lavedán, M. I. (2005). La teoría social de Ortega y Gasset: los usos, Madrid, Biblioteca Nueva.

García-Gómez, J. (2009). Caminos de reflexión. La teoría orteguiana de las ideas y las creencias en el contexto de la Escuela de Madrid, Madrid, Biblioteca Nueva.

Lasaga Medina, J. (2003). José Ortega y Gasset (1883-1955). Vida y filosofía, Madrid, Biblioteca Nueva.

Lorenzo Alquezar, R. (1999). El concepto de creencia en Ortega, UNED, Tesis doctoral inédita.

Marías, J. (1969). La estructura social en Obras VI, Madrid, Revista de Occidente.

Morón Arroyo, C. (1968). El sistema de Ortega y Gasset, Madrid, Ediciones Alcalá.

Ortega y Gasset, J. (2004-2010). Obras completas, Madrid, Taurus/Fundación José Ortega y Gasset.

San Martín, J. (1998). Fenomenología y cultura en Ortega. Ensayos de interpretación, Madrid, Tecnos.

Recibido: 09/07/2013

Aceptado: 03/02/2014 